\title{
Stercula setigera Del. : effet sur les cultures
}

\author{
Charles Bakhoum, Samba Arona Ndiaye Samba*, Babou Ndour \\ Institut Sénégalais de Recherches Agricoles, Direction des Recherches sur les Productions Forestières, \\ BP 2312, Dakar - Hann, Sénégal
}

(Reçue le 12 octobre 1999 ; acceptée le 21 décembre 1999)

\begin{abstract}
Résumé - En zone soudano-sahélienne plusieurs espèces ligneuses sont maintenues dans les champs pour diverses raisons. Leur effet sur les cultures demeure cependant inconnu pour la plupart d'entre elles. Une étude menée au Sénégal a montré que la densité des cultures (arachide, mil et sorgho) augmente avec la distance au tronc de Sterculia setigera. La hauteur du sorgho, l'étalement de l'arachide, sa biomasse en gousses ainsi que les biomasses en épis et en tiges de mil et de sorgho augmentent également avec la distance au tronc. Les biomasses en épis (mil et sorgho) et en gousses d'arachide demeurent plus élevées sous le couvert des arbres émondés. Ces résultats révèlent ainsi un effet dépressif de $S$. setigera sur les cultures associées.
\end{abstract}

Sterculia / interactions arbre-cultures / arachide / mil / sorgho

Abstract - Sterculia setigera Del.: effects on crops. In sub-Saharian Africa various tree species are maintained in crop fields by farmers for many reasons, even though their effects on crop yield remain unknown for most of them. A study in Senegal has shown that crop density (peanut, millet and sorghum) increased as distance from Sterculia setigera increased. Sorghum height, peanut plant crown diameter, biomass of peanut pods, millet and sorghum stalks and twigs increased as well with increasing distance from the tree. Under the crown of pruned trees millet and sorghum stalk biomass and peanut pod biomass were higher than under non-pruned trees. Therefore, these results show a depressive effect of $S$. setigera on crop yield.

Sterculia / tree-crop interactions / peanut / millet / sorghum

\section{INTRODUCTION}

Au Sénégal, Sterculia setigera (ou mbepp) forme un parc agroforestier dans le bassin arachidier où l'espèce est associée aux cultures dans un arrangement spatial dispersé. L'espèce, de la famille des Sterculiacées, peut dépasser $35 \mathrm{~m}$ de haut [14]. Elle se rencontre surtout en zone soudano-guinéenne [9] et colonise les sols superficiels et gravillonnaires. Elle joue un rôle économique important par la production de la gomme karaya très utilisée dans l'alimentation humaine. Toutefois, des résultats d'enquêtes menées en milieu rural [12] font état d'un effet dépressif de l'espèce sur les cultures associées. À ce jour, aucune étude agronomique n'a confirmé ces résultats. Les observations conduites dans ce présent travail avaient pour objet de quantifier l'effet dépressif des arbres sur l'arachide, le mil et le sorgho en établissant le gradient des composantes du rendement de ces cultures en fonction de la distance au tronc des arbres et de l'ombrage.

\section{MATÉRIEL ET MÉTHODES}

\subsection{Site de l'étude}

L'étude a eu pour cadre le bassin arachidier du Sénégal, notamment les villages d'Arafat Mbayène et 
Pire, dans la région de Kaolack $\left(14^{\circ}-14^{\circ} 30^{\prime} \mathrm{N}\right.$ et $\left.15^{\circ} 30^{\prime}-16^{\circ} \mathrm{O}\right)$. Cette zone est caractérisée par l'alternance d'une saison sèche de neuf mois (octobre à juin) et d'une saison pluvieuse de trois mois (juillet à septembre), pour une moyenne pluviométrique de 700 à $800 \mathrm{~mm}$ entre 1988 et 1998.

Les sols, de type ferrugineux tropical [8] selon la classification française, présentent une texture grossière, une pauvreté en éléments fins (argiles et limons), en matière organique et en éléments nutritifs. La végétation est caractérisée par une savane arborée composée principalement d'Adansonia digitata, Cordyla pinnata, Pterocarpus erinaceus et $S$. setigera.

\subsection{Effet de la proximité de $S$. setigera et de l'exposition sur les cultures}

L'influence de S. setigera sur le rendement des cultures a été étudiée à Arafat Mbayène avec un échantillon de 89 arbres de diamètre à 1,30 m (DHP) compris entre 45 et $55 \mathrm{~cm}$, situés dans des champs de mil, d'arachide ou de sorgho et éloignés des autres arbres d'une distance
$\mathrm{D} \geq 2 \mathrm{D}_{\mathrm{h}},\left(\mathrm{D}_{\mathrm{h}}=\right.$ diamètre du houppier de l'arbre choisi). Quarante, 32 et 17 arbres ont respectivement été retenus dans les champs de mil, d'arachide et de sorgho. Deux facteurs ont été étudiés: la distance au tronc de S. setigera avec quatre niveaux correspondant aux parcelles P1, P2, P3 et P4 (figure 1) et l'exposition (est, ouest, nord et sud).

Les variables mesurées 3 mois après les semis ont été la hauteur de la plus haute tige pour le mil et le sorgho, la hauteur du plus haut rameau et l'étalement (= diamètre de la partie aérienne) pour l'arachide. Le nombre de sujets par parcelle a également été compté pour chaque culture. Les biomasses $\left(65{ }^{\circ} \mathrm{C}\right.$ pendant $\left.24 \mathrm{~h}\right)$ en épis et tiges de mil et de sorgho ainsi que les biomasses en gousses et totale de l'arachide ont été estimées à la récolte.

\subsection{Effet de l'émondage et de la distance au tronc de $S$. setigera sur les cultures}

L'effet de l'ombrage de S. setigera sur les cultures a été étudié à Pire dans un dispositif à deux facteurs :

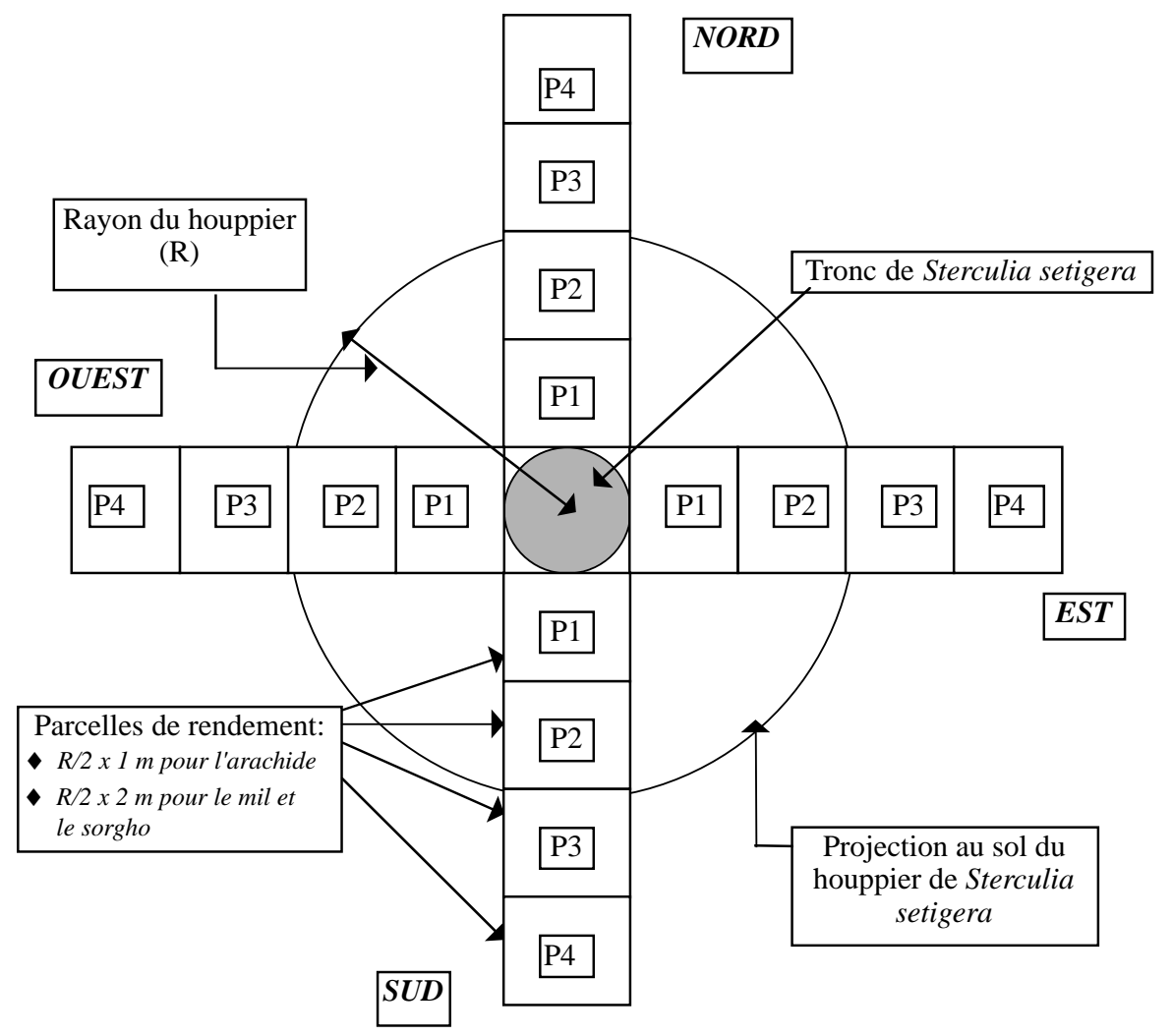

Figure 1. Dispositif expérimental de l'étude sur l'influence de S. setigera sur les cultures. 
l'émondage avec deux niveaux (arbres totalement émondés et arbres non émondés) et la distance au tronc avec 4 niveaux (P1, P2, P3, P4; figure 1). Les parcelles ont été installées à l'ouest des troncs avec respectivement 8,6 et 6 arbres (DHP de 45-55 cm) dans les champs de mil, d'arachide et de sorgho. Pour chaque type de champ, $50 \%$ des arbres choisis ont entièrement été émondés et $50 \%$ laissés intacts. Le nombre de répétitions était ainsi de 4,3 et 3 respectivement dans les champs de mil, d'arachide et de sorgho. Le reste du dispositif était identique au précédent.

\subsection{Analyse des données}

L'analyse statistique a été effectuée par une analyse de variance. Au préalable le test de Bartlett [1] a permis de confirmer l'homogénéité des variances. Le test de Student Newman-Keuls a ensuite été utilisé pour identifier les traitements significativement différents.

\section{RÉSULTATS}

\subsection{Effet de la proximité de Sterculia setigera et de l'exposition sur les cultures}

Nombre de plants par parcelle - La densité des plants d'arachide, de mil et de sorgho augmente avec la distance au tronc de $S$. setigera (figure 2). En général, les différences observées entre les parcelles situées hors du couvert (P3 et P4) de l'espèce sont moins importantes, pour toutes les spéculations, que celles observées entre les parcelles sous le couvert (P1 et P2). L'orientation n'a pas eu d'effet sur la densité des cultures $(P<0,109)$.

Croissance des cultures - Après 3 mois, la croissance en hauteur de l'arachide n'a pas varié en fonction de la distance au tronc de $S$. setigera (figure $3 a$ ). Par contre, celle du mil ou du sorgho est d'autant plus importante que la distance au tronc est importante: $\mathrm{P} 4>\mathrm{P} 3>\mathrm{P} 2>$ $\mathrm{P} 1$ (figures $3 b$ et $3 c$ ). L'étalement de l'arachide augmente également avec la distance au tronc $(P<0,006)$ comme suit: $\mathrm{P} 4=\mathrm{P} 3>\mathrm{P} 2>\mathrm{P} 1$ (figure 4).

Biomasse des cultures - Les biomasses en épis (figures $5 a$ et $5 b$ ) et en tiges (figures $6 a$ et $6 b$ ) de mil et de sorgho sont plus élevées lorsque la distance au tronc est plus grande. Pour l'arachide, l'effet de la proximité de $S$. setigera et celui de l'orientation sont significatifs sur la biomasse en gousses et sur la biomasse totale (fanes + gousses). Celle-ci augmente ainsi d'une part avec la distance au tronc $(P=0,0013$; figure $6 c)$ et elle est d'autre part plus élevée au sud plus exposé qu'à l'est, à l'ouest ou au nord $(P=0,042)$. Les différences entre ces trois dernières expositions n'ont cependant pas été significatives (figure 6d).

\subsection{Effet de l'émondage et la distance au tronc de $S$. setigera sur les cultures}

L'interaction entre l'émondage et la distance au tronc de $S$. setigera est significative pour la biomasse en épis de mil $(P=0,0001)$ et de sorgho $(P=0,0001)$ et pour la biomasse en gousses d'arachide $(\mathrm{P}=0,0001)$. Ces biomasses plus élevées sous le couvert des sujets émondés augmentent également avec la distance au tronc (Figures $7 a, 7 b$ et $7 c$ ). Toutefois, la différence (en \%) entre le rendement moyen des parcelles P1, P2, P3 et P4 pour les arbres émondés et le rendement moyen des mêmes parcelles à proximité des arbres non émondés est moins élevée pour les gousses d'arachide $(-51 \%)$ que pour les épis de mil $(-61 \%)$ ou de sorgho $(-67 \%)$.

\section{DISCUSSION}

Quel que soit le type de culture, la densité des plants est réduite à proximité de $S$. setigera. Il est probable que le taux de germination des semences soit plus bas sous l'influence de l'ombrage qu'en dehors du houppier. Dhawan et al. [5] ont en effet noté une réduction du taux de germination du sorgho et une inhibition de la croissance et de la production de matière sèche du maïs sous l'effet de l'ombrage de Eucalyptus tereticornis. Schutz [13] a également observé une forte réduction de la germination de six espèces du genre Carex sous un couvert dense par rapport au découvert. Il est aussi possible que les parcelles à proximité de $S$. setigera soient plus exposées aux piétinements et à d'autres perturbations du fait qu'elles servent souvent d'abris aux paysans à la recherche d'ombre durant les pauses.

Les biomasses en gousses d'arachide ainsi que les biomasses en épis et en tiges de mil et de sorgho augmentent également avec la distance au tronc de $S$. setigera, donc dans le même sens que le gradient de la lumière. Samba [10] a également observé une tendance similaire de la biomasse des grains de mil à proximité de Cordyla pinnata. Gentle et Duggin [7] ont noté dans une forêt sèche d'Australie qu'une réduction de la couverture végétale (réduction de l'ombrage) avait permis d'augmenter la production de biomasse de Lantana camara de $140 \%$. Par contre, Diakité [6] a plutôt noté une diminution de la biomasse aérienne du sorgho avec l'augmentation de la distance au tronc de Butyrospermum paradoxum. 

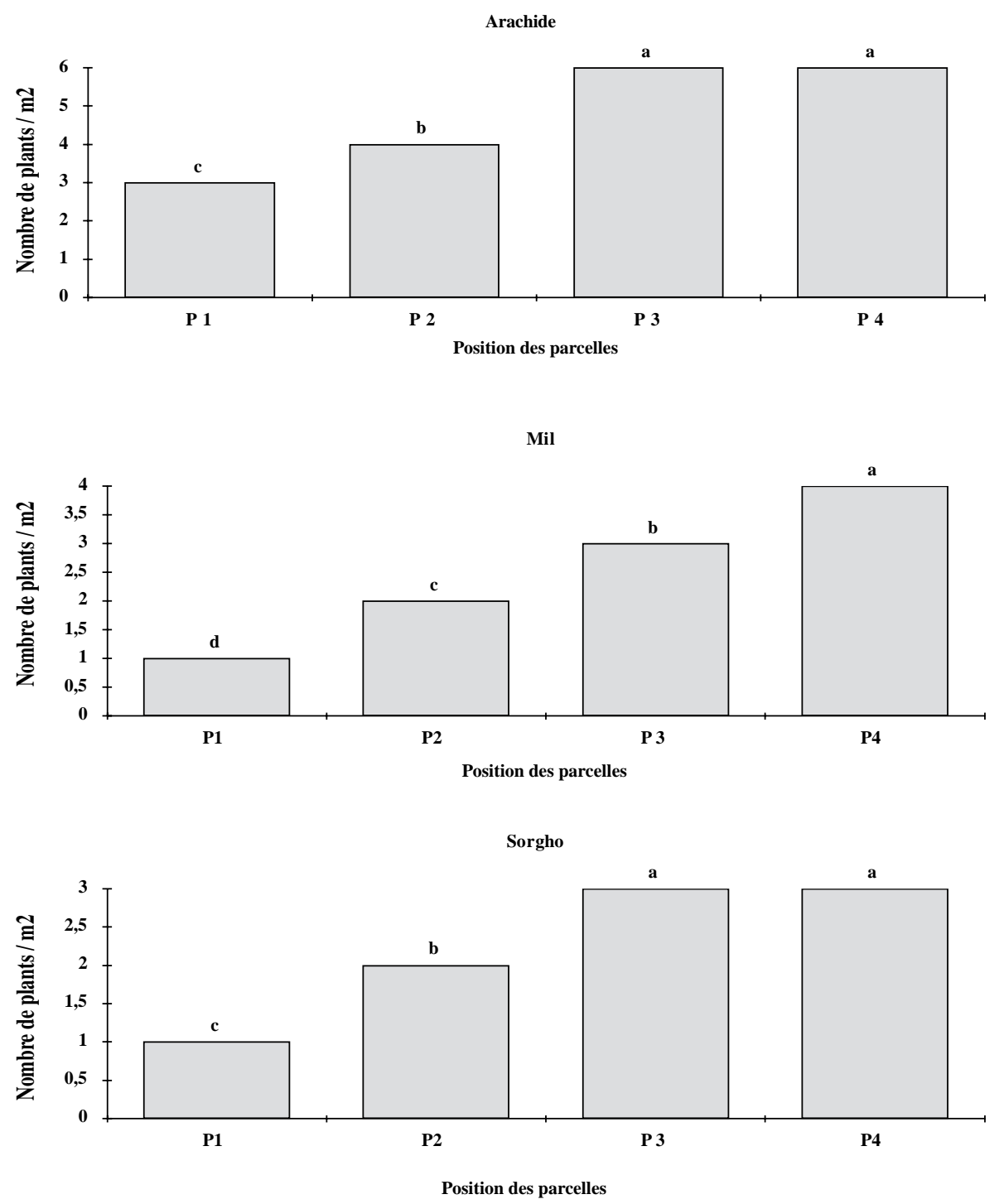

Figure 2. Nombre de plants d'arachide $(P<0,0001)$, de mil $(P<0,0001)$, et de sorgho $(P<0,0001)$ en fonction de la distance au tronc de S. setigera 3 mois après les semis, P1, P2 étant les parcelles sous le couvert de l'espèce et $\mathrm{P} 3$ et $\mathrm{P} 4$ celles en dehors du couvert.

Les résultats de l'étude montrent que la biomasse totale de l'arachide est plus élevée au sud (moins ombragé) et que les biomasses en épis (pour le mil et le sorgho) et en gousses (pour l'arachide) sont plus élevées sous le couvert des sujets émondés (plus de lumière) que sous le couvert des sujets non émondés.

L'ombrage de $S$. setigera exerce ainsi un effet dépressif sur certaines variables du mil, du sorgho et de l'arachide, ce qui contribue à réduire les rendements. Cathey et Campbell [2] ont en effet constaté qu'une diminution de l'intensité du rayonnement utile à la pho- tosynthèse entraîne une baisse de la production de la plante. L'arachide (plante à $\mathrm{C}_{3}$ ) se comporte toutefois mieux que le mil et le sorgho (plantes à $\mathrm{C}_{4}$ ) sous le couvert des arbres.

De plus, la concurrence pour l'eau et les éléments nutritifs peut contribuer à accentuer cette réduction des rendements à proximité de S. setigera. Chopart [3], [4] a noté d'une part que $S$. setigera développe un enracinement peu profond qui exploite la totalité ou une partie des horizons pédologiques de la plupart des espèces cultivées et que le système racinaire du sorgho est d'autre 
Figure 3a. Hauteur des plants $(\mathrm{cm})$ d'arachide $(P=0,765)$ en fonction de la distance au tronc de $S$. setigera 3 mois après les semis

Figure 3b. Hauteur des plants $(\mathrm{cm})$ de mil $(P=0,039)$ en fonction de la distance au tronc de $S$. setigera 3 mois après les semis.

Figure 3c. Hauteur des plants $(\mathrm{cm})$ de sorgho $(P=0,002)$ en fonction de la distance au tronc de $S$. setigera 3 mois après les semis.

Figure 4. Diamètre du houppier de l'arachide $(P<0,006)$ en fonction de la distance au tronc de $S$. setigera 3 mois après les semis.

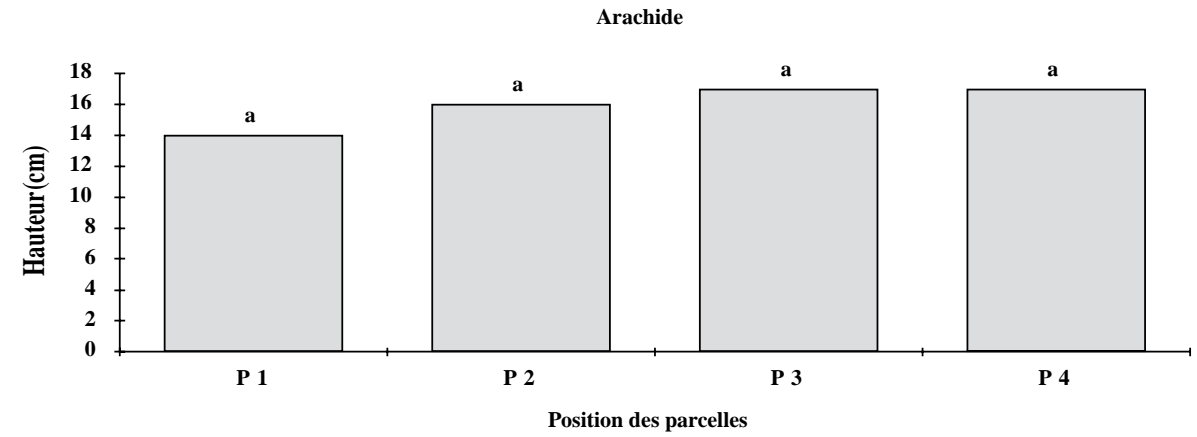

Mil

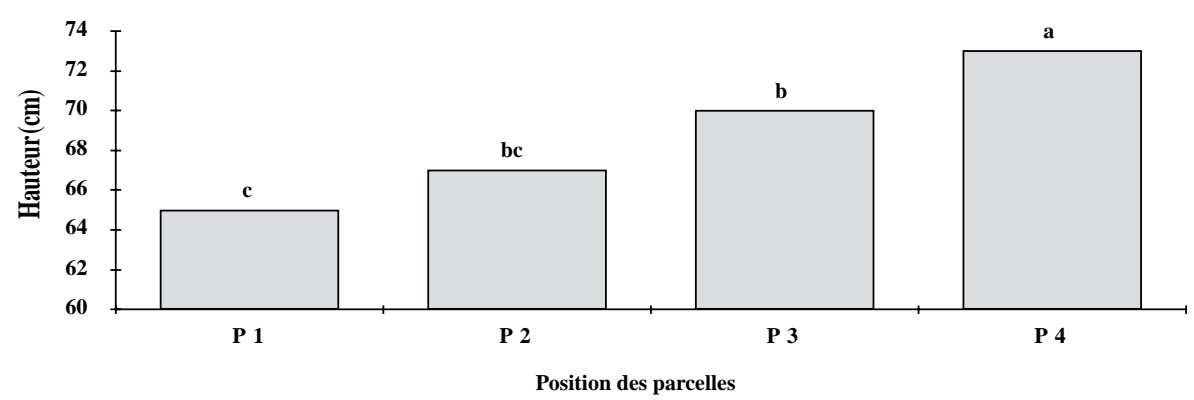

Sorgho
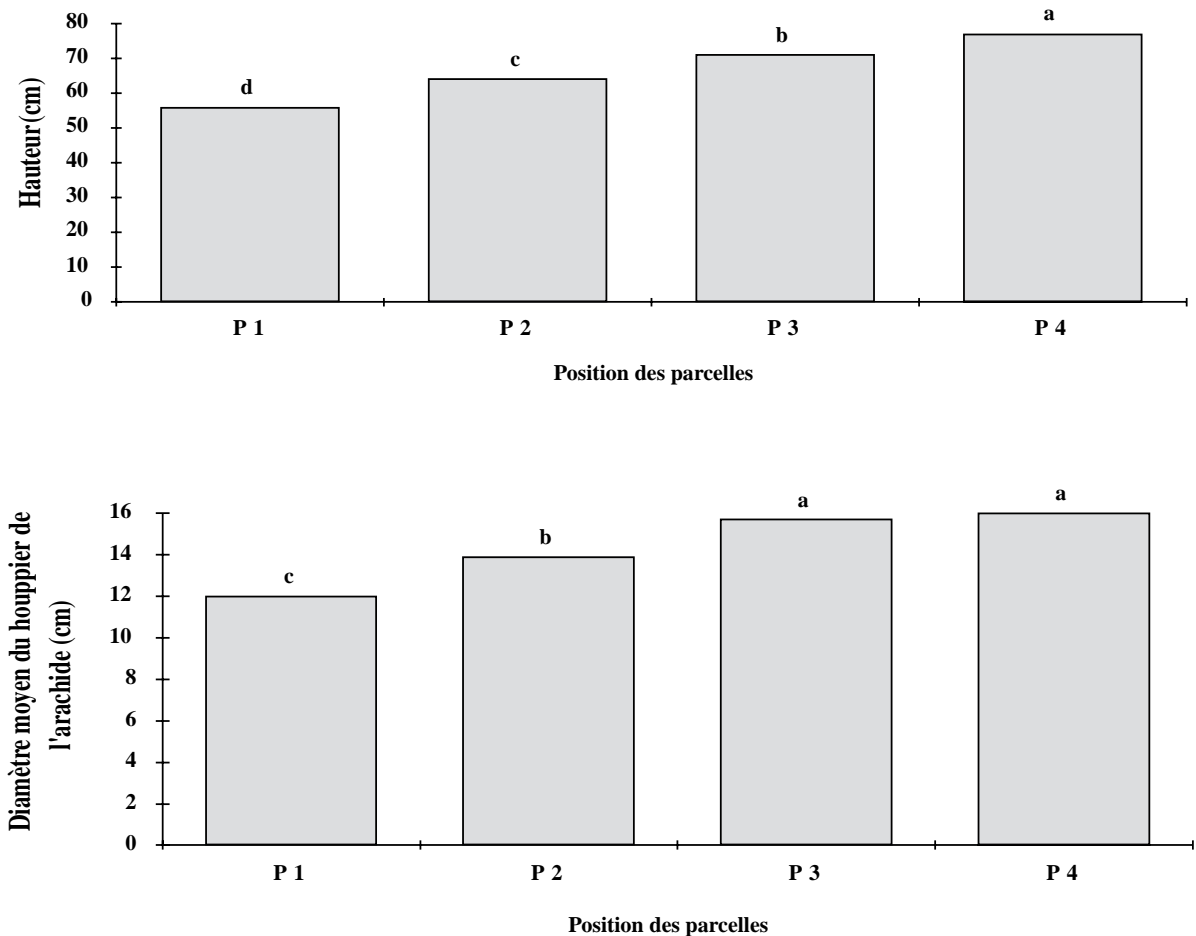
Figure 5a. Biomasse en épis de mil $(P=0,019)$ en fonction de la distance au tronc de $S$. setigera.

Figure 5b. Biomasse en épis de sorgho $(P=0,0022)$ en fonction de la distance au tronc de $S$. setigera.

Figure 6a. Biomasse des tiges de mil $(P=0,041)$ en fonction de la distance au tronc de $S$. setigera.

Figure 6b. Biomasse des tiges de sorgho $(P=0,033)$ en fonction de la distance au tronc de S. setigera.
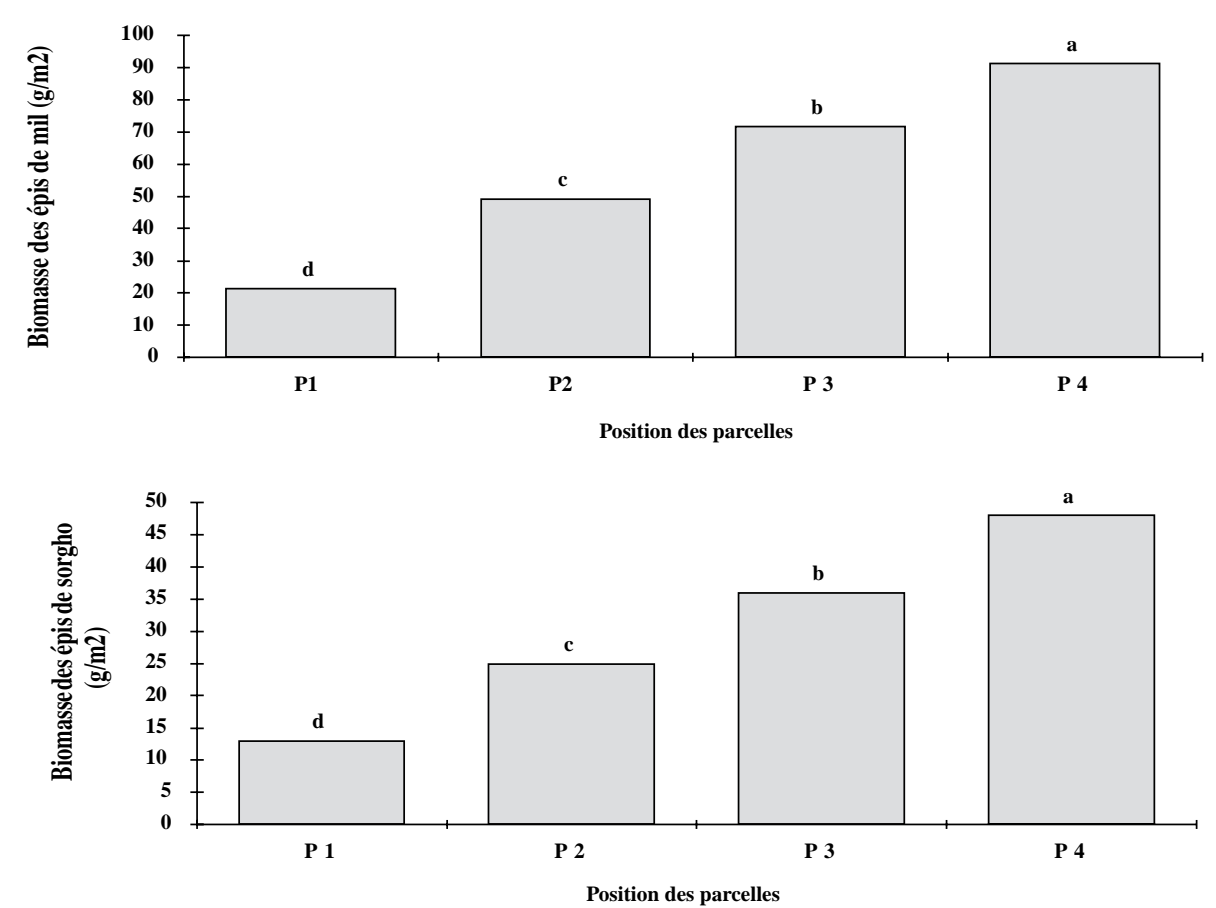

Mil

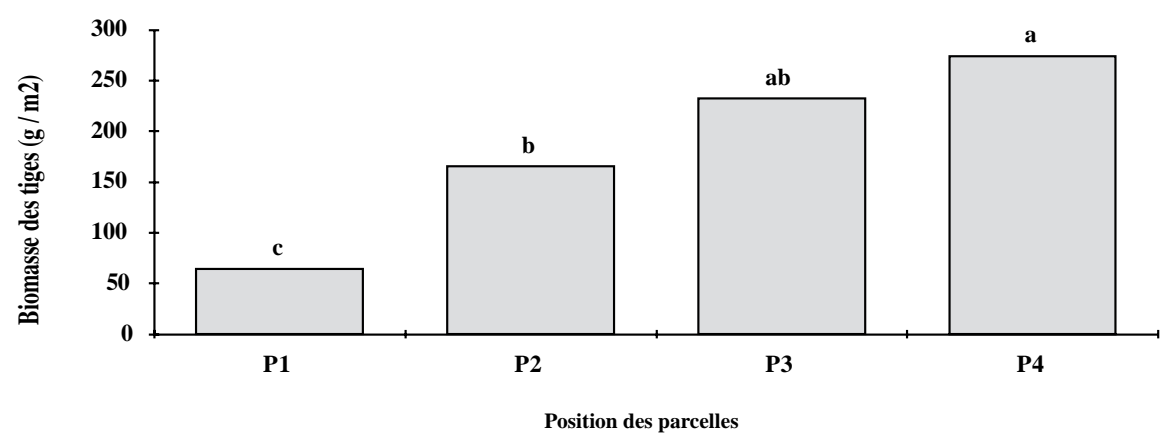

Sorgho

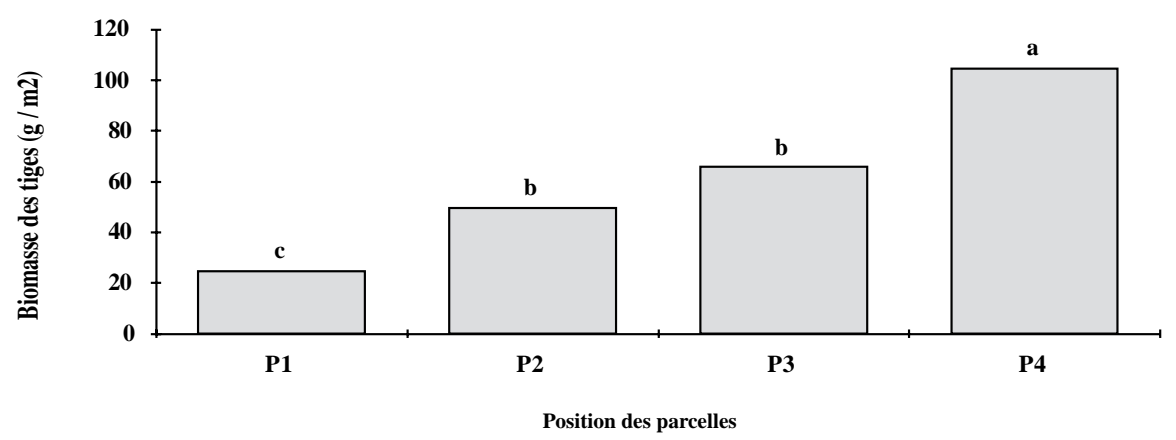


Figure 6c. Biomasse totale (gousses + fanes) d'arachide $(P=0,0013)$ en fonction de la distance au tronc de $S$. setigera.

Figure 6d. Biomasse totale (gousses + fanes) d'arachide $(P=0,042)$ en fonction de l'orientation par rapport au tronc de S. setigera.

Figure 7a. Biomasse en épis de mil $(P=0,0001)$ en fonction de la proximité de $S$. setigera et de l'émondage.

Figure 7b. Biomasse en épis de sorgho $(P=0,0001)$ en fonction de la proximité de $S$. setigera et de l'émondage.
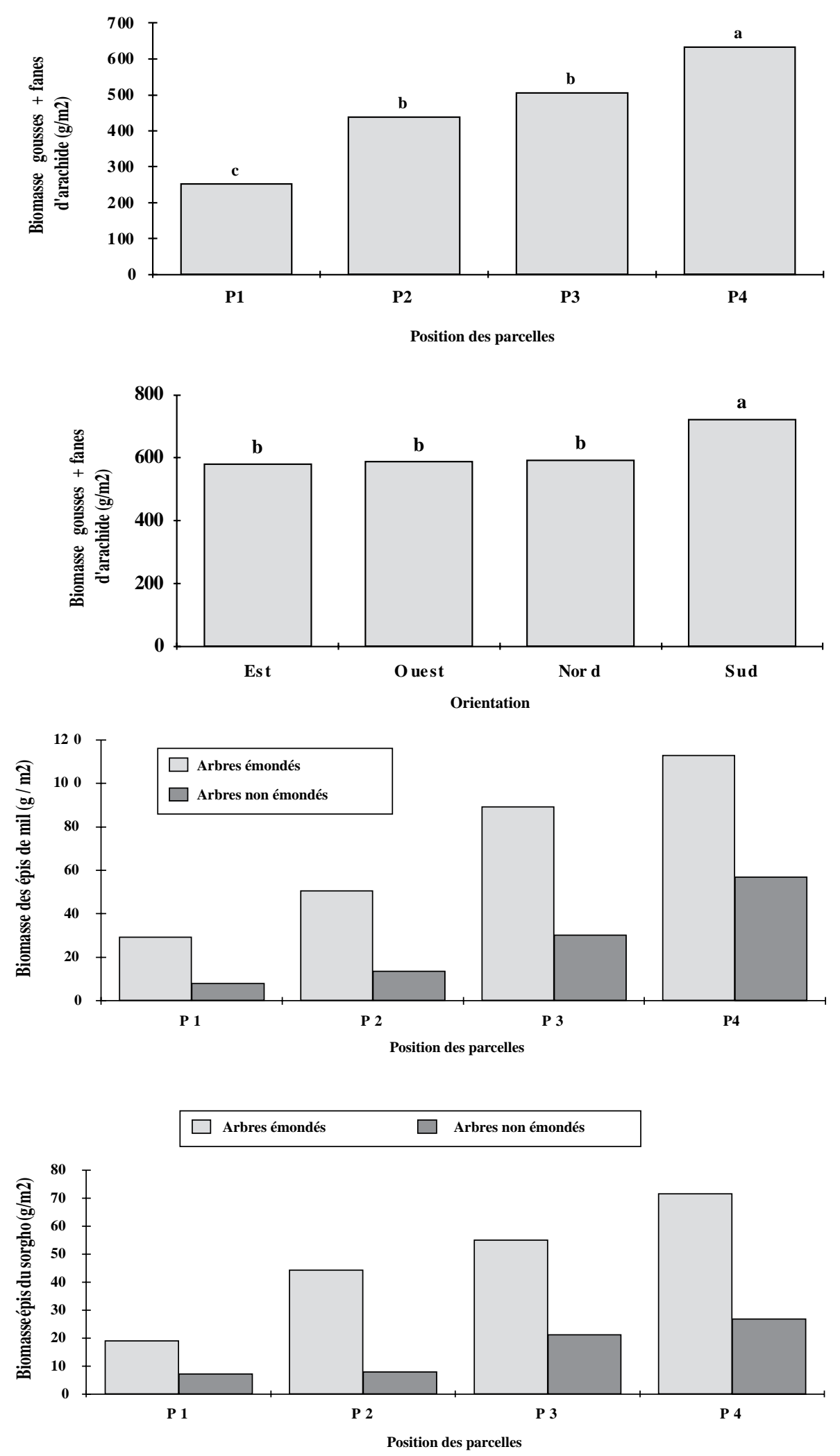
Figure 7c. Biomasse en gousses d'arachide $(P=0,0001)$ en fonction de la proximité de $S$. setigera et de l'émondage.

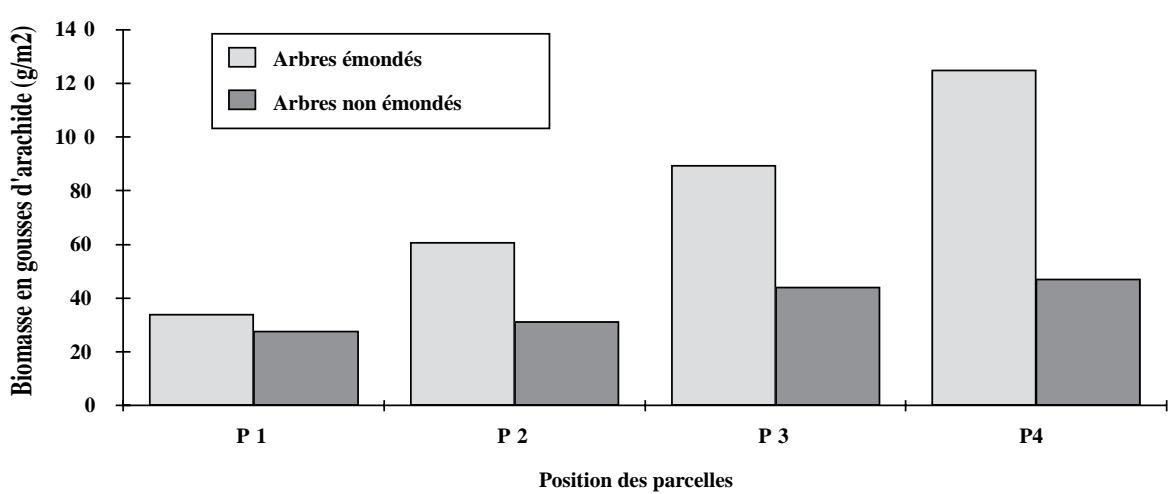

part plus superficiel que celui des autres cultures. La plus forte réduction des rendements sous le houppier de $S$. setigera observée pour le sorgho pourrait ainsi s'expliquer par une concurrence entre l'arbre et la culture dans tout le profil cultural, contrairement à l'arachide et au mil pour qui seulement une partie du profil cultural semble être exploitée par S. setigera.

Samba [11] a par ailleurs observé, dans un essai biologique, une réduction de la biomasse totale de l'arachide suite à des apports importants de litière foliaire de Cordyla pinnata. Selon l'auteur l'hypothèse de la présence d'effets allélopathiques qui inhibent la production de la culture ne doit pas être exclue. Il est également probable que $S$. setigera émette des substances toxiques qui soient responsables de la baisse de production des cultures à sa proximité.

Ainsi la réduction des performances des cultures associées pourrait être due à l'interaction de plusieurs facteurs dont: l'ombrage du mbepp, les propriétés physiques, chimiques et biologiques du sol (modifiées par l'arbre) et l'architecture racinaire de S. setigera.

\section{CONCLUSION}

S. setigera est une espèce de parc qui joue un rôle économique, alimentaire et pastoral très important pour les populations rurales dans le bassin arachidier. L'étude montre cependant que l'espèce a un effet dépressif sur les cultures étudiées. Ces effets sont plus importants sur les céréales, plus exigeantes en lumière que sur l'arachide. Des études complémentaires sont cependant nécessaires pour confirmer les résultats obtenus. Il serait ainsi intéressant d'effectuer des mesures sur d'autres variables comme le taux de photosynthèse et le potentiel hydrique des cultures en fonction de la distance au tronc de $S$. setigera ainsi que des mesures de fertilité et de bilan hydrique du sol en fonction de la distance au tronc de l'arbre pour étudier les relations entre ces différentes variables.

Remerciements : Les auteurs remercient le FAC pour son appui financier, la Direction des Eaux, Forêts, Chasse et Conservation des sols du Sénégal qui a fourni les moyens humains, matériel et logistiques nécessaires pour réaliser cette étude et l'ISRA-DRPF pour son appui scientifique.

\section{RÉFÉRENCES}

[1] Bartlett M.S., The use of transformations, Biom 3 (1947) $39-52$.

[2] Cathy H.M., Campbell L.E., Light and lighting systems for horticultural plants, Hortic. Rev. 2 (1980) 491-533.

[3] Chopart J.L., Étude aux champs des systèmes racinaires des principales cultures pluviales au Sénégal (arachide, mil, sorgho, riz pluvial), thèse d'état, Institut National Polytechnique de Toulouse, France, 1980.

[4] Chopart J.L., Étude du système racinaire du mil (Pennisetum typhoides) dans un sol sableux du Sénégal, CNRA-ISRA Bambey, Sénégal, 1981.

[5] Dhawan R.S., Malick R.K., Samunder S., Singh S., Germination and growth of sorghum under eucalyptus hybrid shelterbelt, in: Integrated weed management for sustainable agriculture, Proc. Indian Society of Weed Science International Symposium, Hisar, India, 18-20 November 1993, Vol. II, Indian Society of Weed Science, Haryana, 1993, pp. 52-55.

[6] Diakité T., Concurrence pour l'eau et les éléments nutritifs du sol entre les ligneux et les cultures - Le Karité et le sorgho en zone semi-aride au Mali, thèse de M. Sc., Université Laval, 1995.

[7] Gentle C.B., Duggin J.A., Lantana camara L. invasions in dry rainforest - open forest ecotones: the role of disturbances associated with fire and cattle grazing, Aust. J. Ecol. 22 (1997) 298-306. 
[8] Giffard P.L., L'arbre dans le paysage sénégalais Sylviculture en zone tropicale sèche, tome I, CTFT, Dakar, 1971.

[9] Kerharo J., Adam J.G., Les plantes médicinales et toxiques du Sénégal, Vigot \& Frères, Paris, 1974.

[10] Samba A.N.S., Influence de Cordyla pinnata sur la fertilité d'un sol ferrugineux tropical et sur le mil et l'arachide dans un système agroforestier traditionnel au Sénégal, thèse de Ph.D., Université Laval, 1997.

[11] Samba A.N.S., Effet de la litière de Cordyla pinnata sur les cultures - Approche expérimentale en agroforesterie, Ann. For. Sci. (submitted).
[12] Séne A., Étude Socio-économique des Systèmes à Parcs dans le Bassin Arachidier - Cas de Sterculia setigera et de Cordyla pinnata, mémoire de confirmation, DRPF-ISRA, Dakar, 1994.

[13] Schutz W., Are germination strategies important for the ability of cespitose wetland sedges (Carex) to grow in forests? Can. J. Bot. 75 (1997) 1692-1699.

[14] Von Maydell H.J., Arbres et Arbustes du Sahel - Leurs caractéristiques et leurs utilisations, Office Allemand de la Coopération Technique (GTZ), Eschborn, 1990.

To access this journal online: www.edpsciences.org 